Article

\title{
Comparative Analysis of the Major Chemical Constituents in Salvia miltiorrhiza Roots, Stems, Leaves and Flowers during Different Growth Periods by UPLC-TQ-MS/MS and HPLC-ELSD Methods
}

\author{
Huiting Zeng ${ }^{1,2}$, Shulan $\mathrm{Su}^{1, *}$, Xiang Xiang ${ }^{1}$, Xiuxiu Sha ${ }^{1}$, Zhenhua Zhu ${ }^{1}$, Yanyan Wang ${ }^{1}$, \\ Sheng Guo ${ }^{1}$, Hui Yan ${ }^{1}$, Dawei Qian ${ }^{1}$ and Jinao Duan ${ }^{1, *}$ \\ 1 Jiangsu Collaborative Innovation Center of Chinese Medicinal Resources Industrialization, National and \\ Local Collaborative Engineering Center of Chinese Medicinal Resources Industrialization and Formulae \\ Innovative Medicine, and Key Laboratory of Chinese Medicinal Resources Recycling Utilization, \\ State Administration of Traditional Chinese Medicine, Nanjing University of Chinese Medicine, \\ Nanjing 210023, China; Zenght1991@163.com (H.Z.); xx5326@126.com (X.X.); shaxiu901128@yeah.net (X.S.); \\ 04040416@163.com (Z.Z.); wyy8127@163.com (Y.W.); gsh916@163.com (S.G.); glory-yan@163.com (H.Y.); \\ qiandwnj@126.com (D.Q.) \\ 2 Department of Traditional Chinese Medicine, Jiangxi Province Academy of Traditional Chinese Medicine, \\ Nanchang 330046, China \\ * Correspondence: sushulan1974@163.com (S.S.); duanja@163.com (J.D.); \\ Tel.: +86-25-8581-1916 (S.S.); +86-25-8581-1116 (J.D.)
}

Academic Editor: Derek J. McPhee

Received: 18 April 2017; Accepted: 8 May 2017; Published: 10 May 2017

\begin{abstract}
Salvia miltiorrhiza is a traditional Chinese herbal medicine containing multiple components that contribute to its notable bioactivities. This article investigated the distribution and dynamic changes of chemical constituents in various parts of S. miltiorrhiza from different growth periods. An ultra-high performance liquid chromatography-triple quadrupole mass spectrometer (UPLC-TQ-MS/MS) and high-performance liquid chromatography coupled with evaporative light scattering detector (HPLC-ELSD) methods were developed for accurate determination of 24 compounds (including phenolic acids, flavonoids, triterpenes, and saccharides) in S. miltiorrhiza. The established methods were validated with good linearity, precision, repeatability, stability, and recovery. Results indicated that there were category and quantity discrepancies in different parts of the plant, for the roots mainly contained salvianolic acids and tanshinones, and most of the saccharides are stachyose. In the aerial parts, salvianolic acids, flavonoids, and triterpenes, except the tanshinones, were detected, and the saccharides were mainly monosaccharides. Dynamic accumulation analysis suggested the proper harvest time for S. miltiorrhiza Bunge was the seedling stage in spring, and for the aerial parts was July to August. This study provided valuable information for the development and utilization value of the aerial parts of S. miltiorrhiza and was useful for determining the optimal harvest time of the plant.
\end{abstract}

Keywords: aerial parts; distribution; dynamic changes; harvest time; S. miltiorrhiza Bunge

\section{Introduction}

Salvia miltiorrhiza (SM), a kind of perennial herb that derived from Salvia genus of the Labiatae family, the dry roots and rhizomes are officially listed in the Chinese Pharmacopoeia [1,2], and it is highly valued and one of the most widely used traditional Chinese medicines (TCM) by virtue of its function to "promote blood circulation and remove blood stasis" that was firstly recorded in 
Shennong's Classic of Materia Medica (200-300 AD, Han Dynasty). The remarkable effects of S. miltiorrhiza on treatment of complicated cardiovascular diseases and cerebrovascular diseases have been reported during the past decades [3-5]. Additionally, numerous studies have demonstrated that S. miltiorrhiza possesses a wide range of pharmacological effects, including anti-oxidative, myocardial infarction, anti-fibrotic [6], anti-inflammatory [7], anti-hypertension [8] and anti-neoplastic [9] effects. Studies show that the efficacious and reliable biological activities of $S$. miltiorrhiza, primarily due to its hundreds of chemical components, which were roughly classified into hydrophilic salvianolic acids, include danshensu (1), protocatechuic aldehyde (2), caffeic acid (3), rosmarinic acid (7), lithospermic acid (8), salvianolic acid B (9), salvianolic acid A (10), etc. and diterpenoid tanshinones comprise of tanshinone IIB (11), dihydrotanshinone I (12), cryptotanshinone (13), neocryptotanshione (14), tanshinone I (15), tanshinone IIA (16), miltiradiene (17), miltirone (18), etc. [10-12]. The chemical structures of these reference compounds are shown in Figure 1.

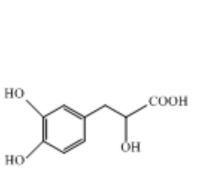

(1)

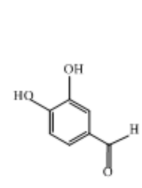

(2)

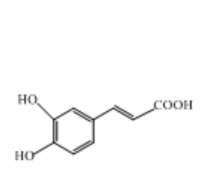

(3)

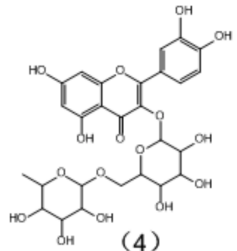

(4)

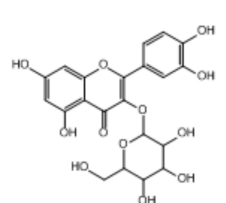

(5)

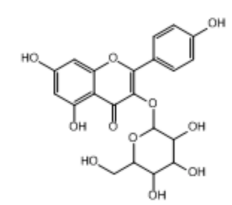

(6)

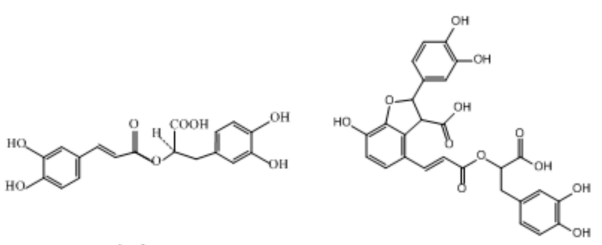

(7)

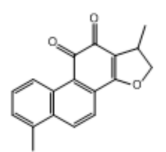

(12)

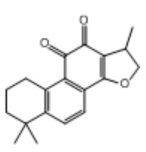

(13)
(8)

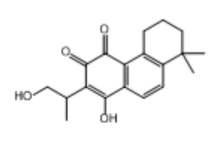

(14)

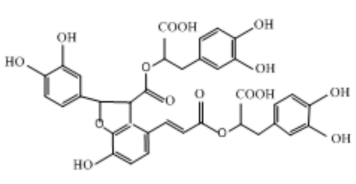

(9)

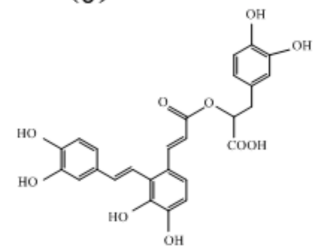

(10)

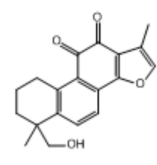

(11)

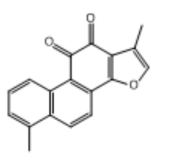

(15)

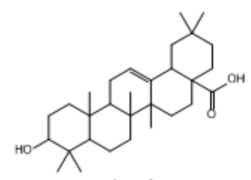

(19)

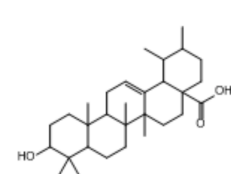

(20)

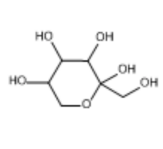

(21)

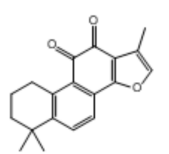

(16)

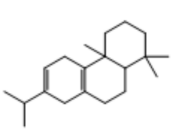

(17)

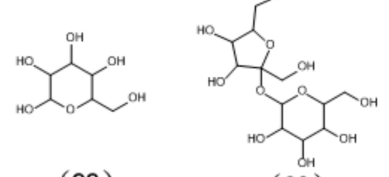

(23)

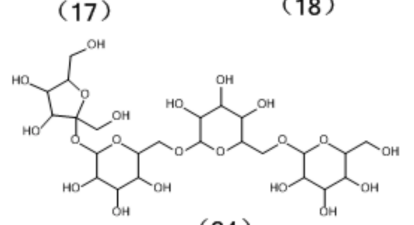

(24)

Figure 1. Chemical structures of 24 reference compounds in S. miltiorrhiza.

On account of the important medicinal value, S. miltiorrhiza, and its relevant preparations, are now drawing worldwide attention with a series of products, such as Danshen injection, Fufang Danshen tablets, tanshinone capsules [13,14], and the Fufang Danshen Dripping Pill, whose phase III clinical study were completed in 2016 [15]. It is estimated that the annual demand of S. miltiorrhiza was about 5000-7000 tons in recent decades, and that the yield of S. miltiorrhiza based on natural resources was insufficient and gradually replaced by artificial cultivation, hence, giving rise to the expansion of planting areas and an increase of production. However, as is well known, the aerial parts of S. miltiorrhiza, whose biomass accounts for about $67 \%$ of the whole plant, was discarded as waste during the root and rhizome harvest, which results in a significant waste of resources and ecological environmental pollution [16]. Therefore, supposing that the aerial parts of S. miltiorrhiza (including the stems, leaves, and flowers) as non-traditional medicinal parts could be fully used, we can make the best of $S$. miltiorrhiza resources [17].

In recent years, studies about the aerial parts of $S$. miltiorrhiza have attracted increasing attention. To the best of our knowledge, the aerial parts of the plant contain abundant biologically-active ingredients [18], and its medicinal value has been cited earlier in the Medical Compliance of the 
Qing Dynasty for "promoting blood circulation and removing blood stasis". Modern studies also showed that the aerial parts of S. miltiorrhiza possess biological activities, such as anti-virus, anti-tumor, anti-inflammation, anti-oxidation, promoting blood circulation and removing blood stasis, anti-atherogenic properties, and so on, and it can be used for the treatment of thrombosis, cardiovascular diseases, for instance for coronary heart disease, and diabetes glucose metabolism disorders [19-23]. Hitherto, the chemical constituents of the aerial parts of S. miltiorrhiza have been discovered and isolated gradually, primarily for the phenolic acids, flavonoids (mainly for rutin (4), isoquercitrin (5), astragalin (6), etc.), triterpenes (mainly for oleanolic acid (19) and ursolic acid (20)), and saccharides (such as fructose (21), glucose (22), sucrose (23), stachyose (24), and so on) [24-26]. The chemical structures of these reference compounds are shown in Figure 1. In a word, it is worthwhile to explore, develop and utilize the aerial parts of the plant.

Until now, there have been reports concerning the growth pattern and optimal collecting period of $S$. miltiorrhiza Bge. $[27,28]$. Nevertheless, there are no published reports about the distribution and dynamic accumulation of various parts of S. miltiorrhiza (roots, stems, leaves, and flowers). In the present study, on the basis of the preliminary experiments in our laboratory $[29,30]$, samples from different parts of S. miltiorrhiza were collected from 2015 to 2016, the sensitive and fast ultra-high performance liquid chromatography-triple quadrupole mass spectrometer (UPLC-TQ-MS/MS) method was developed for simultaneous quantitation of 20 components (seven salvianolic acids, three flavonoids, eight tanshinones, and two triterpenes) in S. miltiorrhiza for the first time, which dramatically simplified the complicated chromatographic separation and identification for multiple components of this plant, and the high-performance liquid chromatography coupled with an evaporative light scattering detector (HPLC-ELSD) method was established for the detection of four saccharides. As a result, our study was attempted to provide a constructive approach for analyzing the changes of these chemical components in separate parts of S. miltiorrhiza at different growth periods. Furthermore, it provided the theoretical basis and scientific evidence for the development and utilization of traditional and non-traditional medicinal parts of S. miltiorrhiza resources. Meanwhile, the established and applied methods in this paper may be useful to the readers, and especially the newcomers, in the research area of $S$. miltiorrhiza resources.

\section{Results and Discussion}

\subsection{Optimization of Mass Spectrometry and Chromatographic Conditions}

In this study, in order to obtain the best mass spectrometry conditions, standard solutions of all of the analytes were tested separately in direct infusion mode by the full-scan MS method in both positive and negative ionization modes. It was found out that flavonoids, tanshinones, and triterpenes had a stronger response in the positive ion mode compared to the negative ion mode. Nevertheless, the tested salvianolic acids showed sensitivity not only in positive mode but also in negative mode, rosmarinic acid, lithospermic acid, salvianolic acid B, and salvianolic acid A obtained from the negative ion mode were higher than that from the positive ion mode, while danshensu, protocatechuic aldehyde, and caffeic acid had better sensitivity and intensity in the positive ion mode, which made it accurate and easy to detect them with lower content levels in S. miltiorrhiza and identify each peak by confirming the molecular ions or quasi-molecular ions. Product ions were automatically chosen according to the stability and ion response by MS. The multiple reaction monitoring (MRM) transitions and parameters of 20 compounds applied in the study are listed in Table 1.

Chromatographic parameters were optimized for achieving a higher separation quality of the chromatogram and reducing the analysis time. As for the mobile phase, comparing with acetonitrile methanol, acetonitrile is a polar aprotic solvent that has been proven the best organic solvent for liquid chromatography, producing narrower peaks in a short analysis time, so that acetonitrile as the organic phase was optimal. In addition, different concentrations of formic acid added in ultrapure water as the aqueous phase was compared in our preliminary test and, finally, $0.1 \%$ formic acid in 
water/acetonitrile were tested for the good separation in determination. The typical chromatograms of 20 compounds are presented in Figure 2. Similarly, for the analysis of saccharides in S. miltiorrhiza, water/acetonitrile was chosen as the preferred mobile phase, and gradient elution was applied during the liquid chromatography process. The injection volume was set at $10 \mu \mathrm{L}$ for samples of roots and leaves, while the injection volume was set to $20 \mu \mathrm{L}$ for stems and flowers due to their lower contents. The flow rate was set at $0.8 \mathrm{~mL} / \mathrm{min}$ and the column temperature was kept at $35^{\circ} \mathrm{C}$. Representative chromatograms of the reference standards are shown in Figure 3.

Table 1. MS/MS detection parameters of 20 compounds in S. miltiorrhiza.

\begin{tabular}{|c|c|c|c|c|c|c|}
\hline Compounds & $t_{\mathrm{R}}(\min )$ & MW & MRM Transitions/SIM & Cone Voltage (V) & Collision Energy (eV) & Ion Mode \\
\hline 1. Danshensu & 2.50 & 198.05 & $199.085>153.028$ & 10 & 6 & $\mathrm{ES}^{+}$ \\
\hline 2. Protocatechuic aldehyde & 3.36 & 138.12 & $138.833>65.075$ & 16 & 20 & $\mathrm{ES}^{+}$ \\
\hline 3. Caffeic acid & 3.89 & 180.04 & $181.030>88.890$ & 12 & 26 & $\mathrm{ES}^{+}$ \\
\hline 5. Isoquercitrin & 4.96 & 464.38 & $465.233>303.104$ & 32 & 22 & $\mathrm{ES}^{+}$ \\
\hline 6. Astragalin & 5.36 & 448.00 & $449.223>287.104$ & 30 & 22 & $\mathrm{ES}^{+}$ \\
\hline 7. Rosmarinic acid & 5.72 & 360.31 & $359.223>197.055$ & 30 & 16 & $\mathrm{ES}^{-}$ \\
\hline 10. Salvianolic acid A & 6.37 & 494.12 & $493.16>295.09$ & 28 & 18 & $\mathrm{ES}^{-}$ \\
\hline 11. Tanshinone IIB & 8.60 & 310.00 & $311.223>293.44$ & 22 & 12 & $\mathrm{ES}^{+}$ \\
\hline 12.Dihydrotanshinone I & 9.22 & 278.30 & $279.224>189.917$ & 28 & 34 & $\mathrm{ES}^{+}$ \\
\hline 13. Cryptotanshinone & 9.71 & 296.35 & $297.16>251.119$ & 36 & 26 & $\mathrm{ES}^{+}$ \\
\hline 14. Neocryptotanshione & 9.75 & 314.38 & $314.968>294.306$ & 86 & 6 & $\mathrm{ES}^{+}$ \\
\hline 15. Tanshinone I & 10.20 & 276.29 & $277.224>249.147$ & 30 & 20 & $\mathrm{ES}^{+}$ \\
\hline
\end{tabular}
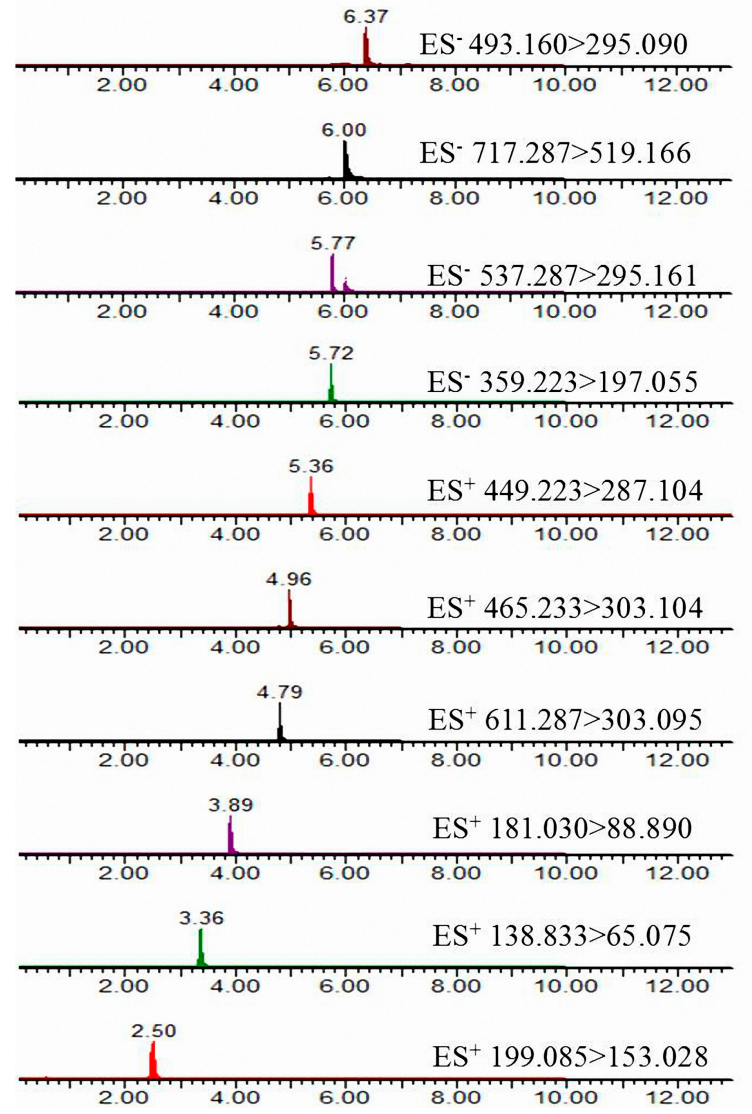

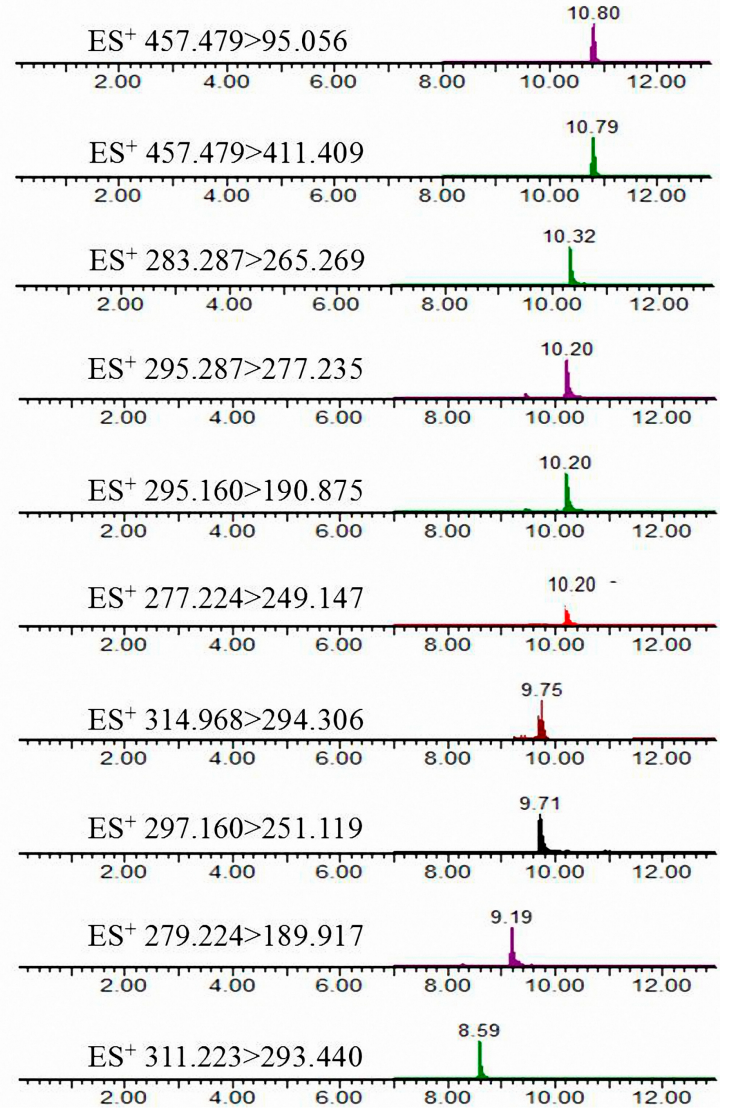

Figure 2. UPLC-TQ-MS/MS chromatogram of 20 compounds in S. miltiorrhiza. 


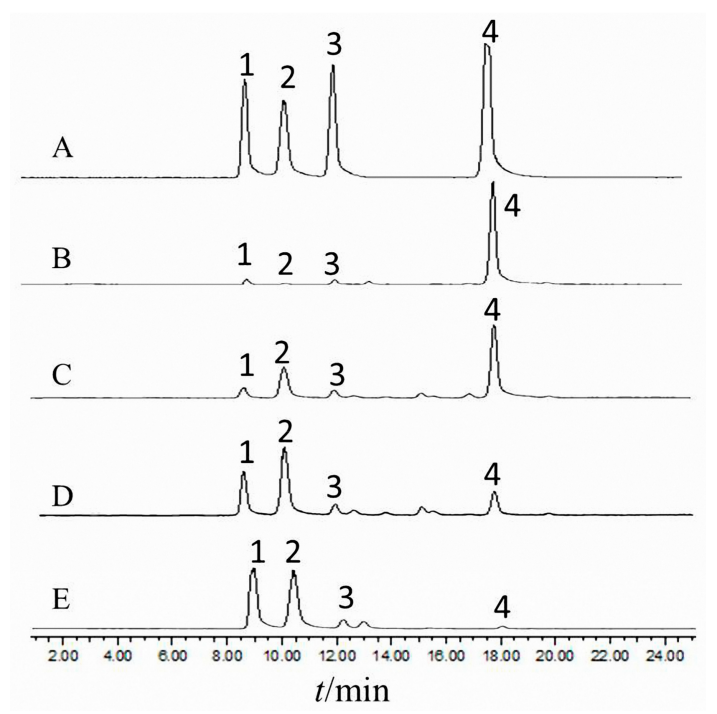

Figure 3. HPLC-ELSD chromatogram of different parts in S. miltiorrhiza. (A) Mixed reference; (B) roots; (C) stems; (D) leaves; and (E) flowers; (1) fructose; (2) glucose; (3) sucrose; (4); stachyose.

\subsection{Method Validation}

The developed UPLC-TQ-MS/MS method for quantitation of salvianolic acids, flavonoids, tanshinones, and triterpenes, and the HPLC-ELSD method for quantitation of saccharides were validated by determining the linearity, LODs, LOQs, precision, repeatability, stability, and recovery. The results are shown in Table 2. All of the marker substances showed good linearity with the determination coefficients $\left(\mathrm{R}^{2}\right)$ ranging from 0.9900 to 0.9998 in a relatively wide concentration range. The results of precision test RSD values of the 24 analytes were less than $4.74 \%$, repeatability and stability RSD values were less than $4.91 \%$, and the overall recoveries were between $98.22 \%$ and $102.34 \%$ for the 24 reference compounds, with RSDs less than $3.61 \%$. As a result, this showed that the established methods were accurate enough for the determination of 24 bioactive components in various parts of S. miltiorrhiza during different harvest times. Therefore, the UPLC-TQ-MS/MS and HPLC-ELSD methods are accurate, precise, and sensitive enough for quantitative evaluation of those salvianolic acids, flavonoids, tanshinones, triterpenes, and saccharides in the massive samples.

\subsection{Distribution Characteristics of Five Kinds of Chemical Constituents in Different Parts of S. miltiorrhiza}

The established UPLC-TQ-MS/MS method was well applied to the simultaneous determination of salvianolic acids (danshensu, protocatechuic aldehyde, caffeic acid, rosmarinic acid, lithospermic acid, salvianolic acid B, and salvianolic acid A), flavonoids (rutin, isoquercitrin, and astragalin), tanshinones (tanshinone IIB, dihydrotanshinone I, cryptotanshinone, neocryptotanshione, tanshinone I, tanshinone IIA, miltiradiene, and miltirone), and the triterpenes included oleanolic acid and ursolic acid. The results (Table 3) show that the main chemical components in roots of S. miltiorrhiza were salvianolic acids and tanshinones, without flavonoids and triterpenes being detected, while in the aerial parts of S. miltiorrhiza, it mainly contained the salvianolic acids, flavonoids, and triterpenes, and the liposoluble tanshinones were not detected. The results of this study are consistent with a previous report [18]. In general, the contents of total salvianolic acids in different parts of S. miltiorrhiza were: root $>$ leaf $>$ flower $>$ stem; the contents of total flavonoids were leaf $>$ flower $>$ stem; and the triterpenes contents were flower $>$ stem $>$ leaf. Salvianolic acids were the common components of aerial parts and underground parts, and the concentration differs from each other at different growth stages. This prompts that salvianolic acids may be primarily biosynthesized in the leaves of S. miltiorrhiza, and meanwhile transferred to the roots by means of stems as the transport organ. It may also synthesize both in the aerial parts and underground parts and transported reciprocally [31]. 
Table 2. Calibration curves, LOD, LOQ, precision, repeatability, stability, and recovery of the 24 references.

\begin{tabular}{|c|c|c|c|c|c|c|c|c|c|c|}
\hline \multirow{2}{*}{ Compounds } & \multirow{2}{*}{ Calibration Curves } & \multirow{2}{*}{$\mathbf{R}^{2}$} & \multirow{2}{*}{$\begin{array}{c}\text { Linear } \\
\text { Range } / \mu g / m L\end{array}$} & \multirow{2}{*}{$\mathrm{LOD} / \mathrm{ng} / \mathrm{mL}$} & \multirow{2}{*}{$\mathrm{LOQ} / \mathrm{ng} / \mathrm{mL}$} & \multirow{2}{*}{$\begin{array}{l}\text { Precision } \\
(\%, n=6)\end{array}$} & \multirow{2}{*}{$\begin{array}{c}\text { Repeatability } \\
(\%, n=6)\end{array}$} & \multirow{2}{*}{$\begin{array}{l}\text { Stability } \\
(\%, n=6)\end{array}$} & \multicolumn{2}{|c|}{ Recovery $(\%, n=3)$} \\
\hline & & & & & & & & & Mean & RSD \\
\hline 1. Danshensu & $Y=1449.8 X-477.58$ & 0.9998 & $1.15 \sim 114.50$ & 55.91 & 186.36 & 3.13 & 3.24 & 4.26 & 101.07 & 2.13 \\
\hline $\begin{array}{l}\text { 2.Protocatechuic } \\
\text { aldehyde }\end{array}$ & $Y=23160 X-1692.6$ & 0.9982 & $0.16 \sim 16.20$ & 7.63 & 25.42 & 3.12 & 4.38 & 3.31 & 99.65 & 3.21 \\
\hline 3. Caffeic acid & $Y=9923.3 X-221.72$ & 0.9981 & $0.19 \sim 19.00$ & 14.55 & 48.50 & 3.33 & 3.72 & 3.36 & 99.86 & 2.21 \\
\hline 4. Rutin & $Y=8337.1 X+17508$ & 0.9986 & $1.01 \sim 101.00$ & 4.05 & 13.50 & 2.97 & 3.59 & 4.29 & 100.79 & 2.43 \\
\hline 5. Isoquercitrin & $Y=6526.3 X+7495.6$ & 0.9996 & $0.93 \sim 92.50$ & 7.05 & 23.50 & 1.12 & 2.87 & 3.62 & 99.09 & 1.99 \\
\hline 6. Astragalin & $Y=18160 X-3328.2$ & 0.9994 & $0.74 \sim 74.00$ & 4.09 & 13.64 & 2.11 & 4.39 & 2.42 & 101.91 & 3.21 \\
\hline 7. Rosmarinic acid & $Y=2132.8 X+4654.1$ & 0.9989 & $1.27 \sim 126.50$ & 22.85 & 76.16 & 1.68 & 3.96 & 1.82 & 98.68 & 2.65 \\
\hline 8. Lithospermic acid & $Y=375.37 X+1307.5$ & 0.9915 & $1.90 \sim 380.00$ & 93.37 & 311.24 & 2.10 & 4.10 & 3.64 & 99.76 & 3.21 \\
\hline 9. Salvianolic acid B & $Y=1292.2 X+4951.3$ & 0.9986 & $1.29 \sim 257.00$ & 66.37 & 221.22 & 1.73 & 3.85 & 1.35 & 101.25 & 1.34 \\
\hline 10. Salvianolic acid A & $Y=4008.9 X+172.38$ & 0.9972 & $0.174 \sim 17.40$ & 33.08 & 110.27 & 1.87 & 3.90 & 3.36 & 99.82 & 2.93 \\
\hline 11. Tanshinone IIB & $Y=133524 X+35,802$ & 0.9967 & $0.07 \sim 7.10$ & 3.12 & 10.40 & 1.73 & 3.21 & 4.91 & 102.23 & 2.36 \\
\hline 12.Dihydrotanshinone I & $Y=232666 X+126,802$ & 0.9972 & $0.13 \sim 12.60$ & 8.20 & 27.33 & 2.86 & 4.55 & 2.43 & 98.22 & 3.25 \\
\hline 13.Cryptotanshinone & $Y=293851 X+578,254$ & 0.9955 & $0.24 \sim 23.60$ & 11.42 & 38.06 & 4.55 & 4.91 & 2.78 & 101.15 & 1.52 \\
\hline 14.Neocryptotanshione & $Y=100.92 X+24.255$ & 0.9976 & $0.19 \sim 18.60$ & 213.67 & 712.29 & 2.86 & 3.15 & 3.12 & 100.18 & 2.83 \\
\hline 15. Tanshinone I & $Y=32735 X+19,739$ & 0.9961 & $0.11 \sim 10.70$ & 40.04 & 133.48 & 2.25 & 3.48 & 3.47 & 99.82 & 3.29 \\
\hline 16.Tanshinone IIA & $Y=355880 X+261,945$ & 0.9942 & $0.113 \sim 11.30$ & 5.24 & 17.47 & 4.74 & 1.19 & 4.26 & 102.34 & 1.58 \\
\hline 17. Miltiradiene & $Y=794169 X+561,247$ & 0.9954 & $0.09 \sim 8.90$ & 2.73 & 9.10 & 4.60 & 4.06 & 4.28 & 100.34 & 3.28 \\
\hline 18. Miltirone & $Y=88227 X+109,759$ & 0.9991 & $0.72 \sim 72.00$ & 4.53 & 15.11 & 1.14 & 3.94 & 4.08 & 101.64 & 2.43 \\
\hline 19. Oleanolic acid & $Y=8484.5 X+6510.4$ & 0.9988 & $0.18 \sim 17.60$ & 14.93 & 49.78 & 3.01 & 4.02 & 3.73 & 99.01 & 1.54 \\
\hline 20. Ursolic acid & $Y=1064.6 X+5087.3$ & 0.9979 & $1.04 \sim 103.50$ & 38.37 & 129.91 & 2.57 & 4.44 & 4.15 & 99.90 & 3.24 \\
\hline 21. Fructose & $Y=1.4383 X+5.879$ & 0.9923 & $32 \sim 3200$ & $4.14 \times 10^{4}$ & $1.38 \times 10^{5}$ & 1.34 & 2.78 & 3.11 & 100.35 & 2.45 \\
\hline 22. Glucose & $Y=1.3669 X+6.6307$ & 0.9937 & 30.54 3054.00 & $2.52 \times 10^{4}$ & $8.39 \times 10^{4}$ & 1.83 & 2.69 & 2.13 & 101.35 & 2.52 \\
\hline 23. Sucrose & $Y=1.2227 X+7.9658$ & 0.9941 & $26.06 \sim 2606.00$ & $3.83 \times 10^{4}$ & $1.28 \times 10^{5}$ & 1.75 & 3.88 & 2.47 & 102.23 & 3.61 \\
\hline 24. Stachyose & $Y=1.2012 X+7.9475$ & 0.9900 & $50.26 \sim 5026.00$ & $2.19 \times 10^{4}$ & $7.31 \times 10^{4}$ & 1.92 & 2.31 & 3.23 & 99.43 & 1.35 \\
\hline
\end{tabular}


Table 3. Contents of salvianolic acids, tanshinones, flavonoids, and triterpenes in different growth periods of different parts in S.miltiorrhiza (mg/g).

\begin{tabular}{|c|c|c|c|c|c|c|c|c|c|c|c|c|}
\hline \multirow{2}{*}{ Harvest Year } & \multirow{2}{*}{ Harvest Date } & \multicolumn{4}{|c|}{ Total Phenolic Acids } & \multirow{2}{*}{$\begin{array}{c}\text { Total Tanshinones } \\
\text { Roots }\end{array}$} & \multicolumn{3}{|c|}{ Total Flavonoids } & \multicolumn{3}{|c|}{ Total Triterpenes } \\
\hline & & Roots & Stems & Leaves & Flowers & & Stems & Leaves & Flowers & Stems & Leaves & Flowers \\
\hline \multirow{5}{*}{2016} & 4.01 & 45.30 & $\mathrm{n}$ & 18.25 & $\mathrm{n}$ & 5.90 & $\mathrm{n}$ & 1.53 & $\mathrm{n}$ & $\mathrm{n}$ & 1.38 & $\mathrm{n}$ \\
\hline & 4.15 & 39.17 & 22.05 & 22.66 & $\mathrm{n}$ & 5.81 & 0.17 & 3.17 & $\mathrm{n}$ & 2.17 & 1.47 & $\mathrm{n}$ \\
\hline & 4.29 & 65.98 & 20.30 & 35.24 & 44.96 & 4.21 & 0.75 & 4.54 & 4.02 & 2.42 & 1.45 & 2.15 \\
\hline & 5.14 & 62.23 & 19.25 & 36.40 & 40.81 & 6.09 & 0.47 & 5.53 & 3.85 & 2.39 & 1.31 & 2.52 \\
\hline & 5.30 & 61.42 & 22.80 & 37.90 & 25.99 & 7.16 & 0.86 & 5.16 & 3.82 & 2.66 & 1.70 & 5.82 \\
\hline \multirow{12}{*}{2015} & 6.08 & 62.11 & 21.17 & 39.49 & 31.36 & 2.85 & 0.71 & 4.84 & 2.00 & 2.63 & 2.24 & 5.91 \\
\hline & 6.18 & 46.61 & 19.76 & 37.00 & 37.42 & 3.32 & 0.78 & 5.97 & 2.98 & 3.43 & 2.15 & 5.00 \\
\hline & 6.25 & 44.60 & 23.55 & 38.16 & 21.97 & 1.24 & 0.62 & 3.79 & 1.05 & 1.57 & 1.72 & 5.33 \\
\hline & 7.04 & 54.29 & 30.58 & 38.81 & $\mathrm{n}$ & 1.99 & 1.01 & 5.40 & $\mathrm{n}$ & 2.11 & 1.87 & $\mathrm{n}$ \\
\hline & 7.14 & 43.48 & 28.22 & 20.53 & $\mathrm{n}$ & 4.44 & 0.45 & 4.11 & $\mathrm{n}$ & 2.25 & 1.85 & $\mathrm{n}$ \\
\hline & 7.23 & 60.33 & 33.57 & 46.68 & $\mathrm{n}$ & 5.06 & 0.92 & 5.63 & $\mathrm{n}$ & 2.12 & 1.77 & $\mathrm{n}$ \\
\hline & 8.12 & 62.45 & 34.74 & 44.14 & $\mathrm{n}$ & 3.61 & 0.54 & 2.68 & $\mathrm{n}$ & 1.84 & 1.64 & $\mathrm{n}$ \\
\hline & 8.26 & 52.59 & 29.58 & 37.91 & $\mathrm{n}$ & 4.29 & 0.78 & 1.15 & $\mathrm{n}$ & 2.06 & 1.47 & $\mathrm{n}$ \\
\hline & 9.19 & 52.91 & 36.82 & 42.25 & $\mathrm{n}$ & 4.68 & 0.60 & 1.93 & $\mathrm{n}$ & 2.06 & 1.51 & $\mathrm{n}$ \\
\hline & 10.19 & 58.34 & $\mathrm{n}$ & $\mathrm{n}$ & $\mathrm{n}$ & 3.74 & $\mathrm{n}$ & $\mathrm{n}$ & $\mathrm{n}$ & $\mathrm{n}$ & $\mathrm{n}$ & $\mathrm{n}$ \\
\hline & 11.29 & 46.69 & $\mathrm{n}$ & $\mathrm{n}$ & $\mathrm{n}$ & 5.37 & $\mathrm{n}$ & $\mathrm{n}$ & $\mathrm{n}$ & $\mathrm{n}$ & $\mathrm{n}$ & $\mathrm{n}$ \\
\hline & 12.30 & 58.73 & $\mathrm{n}$ & $\mathrm{n}$ & $\mathrm{n}$ & 4.26 & $\mathrm{n}$ & $\mathrm{n}$ & $\mathrm{n}$ & $\mathrm{n}$ & $\mathrm{n}$ & $\mathrm{n}$ \\
\hline
\end{tabular}

The " $\mathrm{n}$ " represents without harvest. 
Table 4. Contents of saccharides in different growth periods of different parts in S. miltiorrhiza $(\mathrm{mg} / \mathrm{g})$.

\begin{tabular}{|c|c|c|c|c|c|c|c|c|c|c|c|c|c|}
\hline \multirow{2}{*}{ Harvest Year } & \multirow{2}{*}{ Harvest Date } & \multicolumn{4}{|c|}{ Glucose } & \multicolumn{4}{|c|}{ Sucrose } & \multicolumn{4}{|c|}{ Stachyose } \\
\hline & & Roots & Stems & Leaves & Flowers & Roots & Stems & Leaves & Flowers & Roots & Stems & Leaves & Flowers \\
\hline \multirow{5}{*}{2016} & 4.01 & - & $\mathrm{n}$ & 11.10 & $\mathrm{n}$ & 3.06 & $\mathrm{n}$ & - & $\mathrm{n}$ & 229.86 & $\mathrm{n}$ & 1.62 & $\mathrm{n}$ \\
\hline & 4.15 & 2.40 & 41.51 & 19.51 & $\mathrm{n}$ & 2.08 & 1.23 & - & $\mathrm{n}$ & 131.03 & 3.79 & 2.91 & $\mathrm{n}$ \\
\hline & 4.29 & - & 31.43 & 46.06 & 41.30 & 7.19 & 1.60 & 2.01 & 4.50 & 159.14 & 2.03 & 5.55 & 0.81 \\
\hline & 5.14 & - & 9.34 & 46.23 & 52.03 & 3.98 & 0.68 & 2.28 & 1.58 & 129.43 & 3.32 & 4.72 & 0.49 \\
\hline & 5.30 & - & 9.12 & 40.68 & 17.77 & 2.22 & - & 1.28 & 0.51 & 109.72 & 4.61 & 3.35 & 1.15 \\
\hline \multirow{12}{*}{2015} & 6.08 & 5.75 & 22.62 & 29.50 & 27.03 & 11.54 & 0.53 & 0.78 & 1.15 & 279.50 & 22.05 & - & 2.08 \\
\hline & 6.18 & - & 4.79 & 23.80 & 42.92 & 5.21 & 0.70 & 1.61 & 5.07 & 244.40 & 4.20 & 1.28 & 2.16 \\
\hline & 6.25 & 1.96 & 15.83 & 10.63 & 8.99 & 6.38 & 2.44 & 1.17 & - & 175.97 & 6.38 & 3.81 & 6.69 \\
\hline & 7.04 & 3.69 & 20.31 & 35.48 & $\mathrm{n}$ & 14.47 & 3.06 & 4.46 & $\mathrm{n}$ & 268.33 & 27.69 & 1.85 & $\mathrm{n}$ \\
\hline & 7.14 & 1.74 & 8.64 & 10.53 & $\mathrm{n}$ & 7.41 & 2.41 & 2.42 & $\mathrm{n}$ & 269.66 & 29.24 & - & $\mathrm{n}$ \\
\hline & 7.23 & - & 12.77 & 9.62 & $\mathrm{n}$ & 7.62 & 2.58 & 2.29 & $\mathrm{n}$ & 286.16 & 33.07 & 2.03 & $\mathrm{n}$ \\
\hline & 8.12 & - & 11.60 & 5.39 & $\mathrm{n}$ & 8.63 & 1.73 & 0.76 & $\mathrm{n}$ & 253.15 & 23.93 & 1.61 & $\mathrm{n}$ \\
\hline & 8.26 & 3.37 & 10.88 & 13.98 & $\mathrm{n}$ & 3.73 & 1.35 & 2.10 & $\mathrm{n}$ & 173.98 & 3.64 & 1.22 & $\mathrm{n}$ \\
\hline & 9.19 & 4.79 & 12.40 & 23.64 & $\mathrm{n}$ & 9.84 & 1.26 & 1.07 & $\mathrm{n}$ & 239.77 & 10.98 & 0.98 & $\mathrm{n}$ \\
\hline & 10.19 & 2.38 & $\mathrm{n}$ & $\mathrm{n}$ & $\mathrm{n}$ & 15.41 & $\mathrm{n}$ & $\mathrm{n}$ & $\mathrm{n}$ & 297.15 & $\mathrm{n}$ & $\mathrm{n}$ & $\mathrm{n}$ \\
\hline & 11.29 & - & $\mathrm{n}$ & $\mathrm{n}$ & $\mathrm{n}$ & 11.67 & $\mathrm{n}$ & $\mathrm{n}$ & $\mathrm{n}$ & 299.71 & $\mathrm{n}$ & $\mathrm{n}$ & $\mathrm{n}$ \\
\hline & 12.30 & 3.70 & $\mathrm{n}$ & $\mathrm{n}$ & $\mathrm{n}$ & 22.70 & $\mathrm{n}$ & $\mathrm{n}$ & $\mathrm{n}$ & 275.49 & $\mathrm{n}$ & $\mathrm{n}$ & $\mathrm{n}$ \\
\hline
\end{tabular}


The HPLC-ELSD method was applied for saccharide analysis of the samples, and the contents of the four saccharides (fructose, glucose, sucrose, and stachyose) are shown in Table 4, which revealed that the four saccharides exist in each part of $S$. miltiorrhiza, and the contents differ from each other. In the roots of S. miltiorrhiza, the content of stachyose, which possesses a high development value, was significantly higher than other parts of the plant, followed by the stems. Similarly, the disaccharide (sucrose) was detected mostly in the roots, as well; however, the two monosaccharides (fructose and glucose) mainly exist in leaves and flowers. The results revealed that in S. miltiorrhiza, the monosaccharides probably mainly synthetize in organs for photosynthesis, like leaves and flowers, while constituents of disaccharides and polysaccharides may form by dehydration with different kinds of monosaccharides in conducting tissue and vegetative organs, such as stems and roots [32]. Therefore, it can provide guidance for obtaining various saccharide resources from S. miltiorrhiza, as is known to us that saccharides and their derivatives may always be the important components that are responsible for the pharmacological activities of traditional Chinese medicine (TCM), for instance of stachyose, which has been confirmed for regulating gut microbiota and widely used for the development of health care products [33].

\subsection{Dynamic Accumulation of Chemical Components in Roots of S. miltiorrhiza at Different Growth Stages}

There is a certain growth correlation between cells, tissues, and organs in the plants: they are independent of each other and, meanwhile, closely related. In addition, the environmental factors, such as moisture, temperature, and light, show significant influence during plant growth and component accumulation [34]. Results (Figure 4 and Supplementary Materials Table S1) in the present study indicate that the content changing trend of salvianolic acids in roots of S. miltiorrhiza showed an "M" shape, as high as $6.598 \%$; that is to say, the content of salvianolic acids reached the highest in the spring seedling stage (April to May) and the vigorous growth period of aerial parts (July to August). The results are in agreement with an earlier report [27]. With the temperature rising in spring, nutritive material in roots accumulate rapidly to make preparations for sprouting of the aerial parts. Afterwards, vegetative growth and reproductive growth of the aerial parts are in progress because of the high temperature and strong light in July and August, which results in strong photosynthesis in the leaves. As the aboveground parts and roots of plants form an organic combination which is inherently consistent, during the vigorous growth of aboveground parts, the effective components in roots were also accumulated at the highest levels in a year. As for the tanshinones (Table S2), they reached the highest level of $0.716 \%$ at the turning green stage of the next year. Considering the perennial plant as S. miltiorrhiza, content of tanshinones may accumulate in accordance with increased growth years. When it comes to the saccharides in $S$. miltiorrhiza of different growth periods, it contains a decent amount of stachyoses, and the content reached a maximum during the withering period. In general, two novel concerned active ingredients (salvianolic acids and tanshinones) enriched at the germination stage. Consequently, the optimum harvest time of roots from S. miltiorrhiza should be the seedling stage in spring.

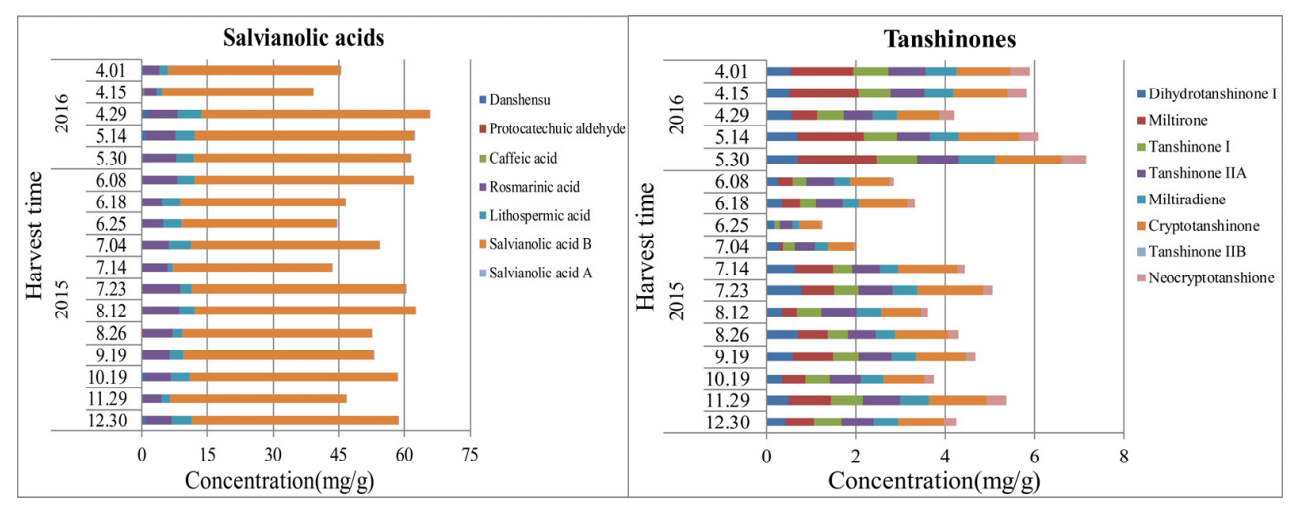

Figure 4. Dynamic accumulation of the contents of salvianolic acids and tanshinones in the roots of S. miltiorrhiza. 
2.5. Dynamic Accumulation of Chemical Components in the Aerial Parts of S. miltiorrhiza (Stems, Leaves, and Flowers) at Different Growth Stages

As the results (Figure 5 and Table S3) indicate, salvianolic acids in the stems of S. miltiorrhiza increased in the whole growth period until mid- to late-September, at which time the aerial parts were tending to wither. However, the content of flavonoids and triterpenes reached the highest levels in mid-June and early July, respectively, probably because of the key enzymes that are involved in the synthesis of flavonoids and triterpenes are at the highest content levels and activity in this period [35]. The contents of monosaccharides (fructose and glucose) showed the highest values in wilting and seedling stages, and disaccharide (sucrose) and oligosaccharide (stachyose) reached their maxima in the vigorous growth period of the aerial parts in July. Further studies are needed to clarify the dynamic accumulation of saccharides in stems.
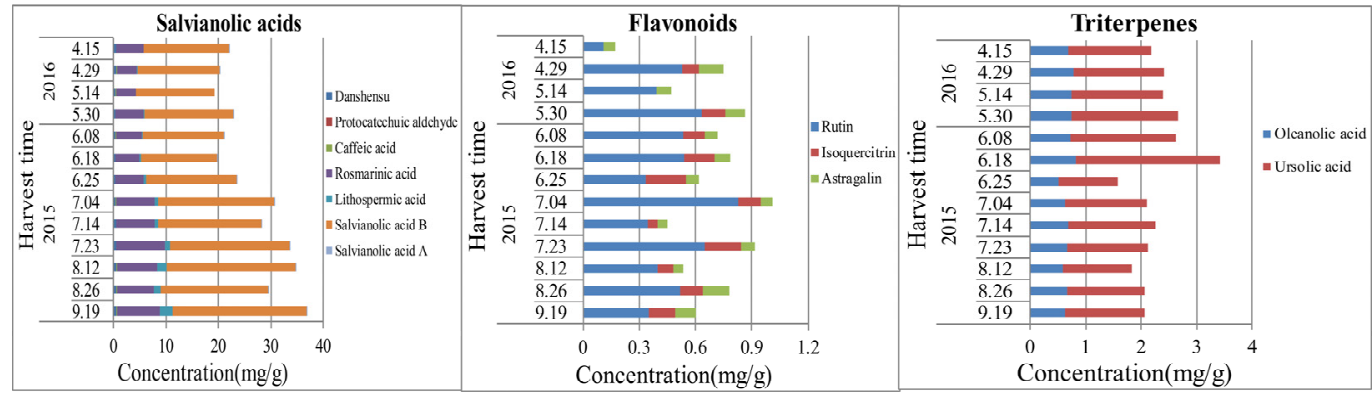

Figure 5. Dynamic accumulation of the contents of salvianolic acids, flavonoids, and triterpenes in the stems of S. miltiorrhiza.

During the whole growth period of the plant, concentrations of salvianolic acids, flavonoids, and triterpenes in leaves increased firstly, and declined afterwards, for the duration of late July to early August, when the temperature and illumination were the highest. For leaves as the synthetic organ, the content of salvianolic acids reached the maximal value, while the maximum accumulation of flavonoids and triterpenes occurs in June. The results are shown in Figure 6 and Table S4. Similarly with the stems, the disaccharide (sucrose) and oligosaccharide (stachyose) contents reached the highest values in July, at which time the photosynthesis was the strongest. Moreover, monosaccharides (fructose and glucose) accumulated to the maximum level in May.

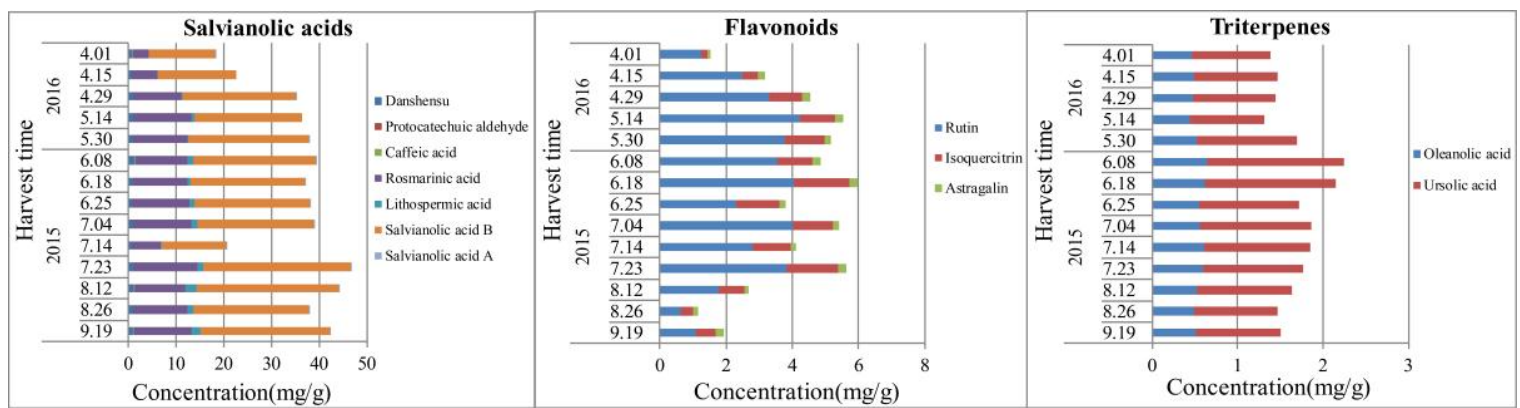

Figure 6. Dynamic accumulation of the contents of salvianolic acids, flavonoids, and triterpenes in the leaves of S. miltiorrhiza.

Analysis of the dynamic accumulation of chemical components in flowers of S. miltiorrhiza at different flowering stages is shown in Figure 7 and Table S5 indicates that the contents of phenolic acids and flavonoids reached the maximum value at the end of April (early bloom stage), while the triterpenes and monosaccharides (fructose and glucose) were high at the end of May to early June 
(full-bloom stage), and the disaccharide (sucrose) and oligosaccharide (stachyose) contents attained the highest levels at the middle to the end of June (late bloom stage). From what is mentioned above, we may come to the conclusion that chemical components of the aerial parts of S. miltiorrhiza were various and abundant, which can be taken into consideration as a new source of salvianolic acids and flavonoids. As a matter of fact, determination of the suitable harvest season of the aerial parts of S. miltiorrhiza should be evaluated according to the actual demand and, supposing salvianolic acid as the main evaluation index, we recommend July and August as the optimum harvest time of the aerial parts of S. miltiorrhiza.

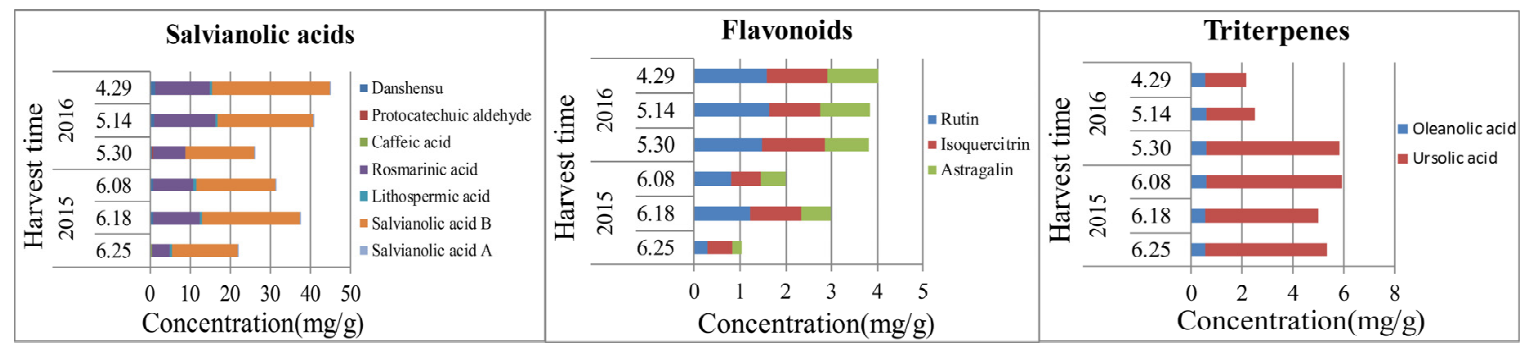

Figure 7. Dynamic accumulation of the contents of salvianolic acids, flavonoids, and triterpenes in the flowers of S. miltiorrhiza.

\section{Materials and Methods}

\subsection{Chemicals and Reagents}

Acetonitrile and formic acid (HPLC grade) were all purchased from Merck (Darmstadt, Germany); deionized water was obtained by a Milli-Q water purification system (Millipore, Billerica, MA, USA). Other chemicals and reagents were of analytical grade. Chemical standards, including protocatechuic aldehyde (2), caffeic acid (3), rosmarinic acid (7), rutin (4), tanshinone IIA (16), and cryptotanshinone (13), were purchased from the National Institute for the Control of Pharmaceutical and Biological Products. Danshensu (1), lithospermic acid (8), salvianolic acid B (9), salvianolic acid A (10), isoquercitrin (5), astragalin (6), dihydrotanshinone I (12), and tanshinone I (15) were purchased from Putian Tongchuang Biotechnology Co. Ltd. (Beijing, China). Tanshinone IIB (11), neocryptotanshione (14), miltiradiene (17), and miltirone (18) were purchased from Guyan Industrial Co., Ltd. (Shanghai, China). Oleanolic acid (19) and ursolic acid (20) were purchased from Preferred Biological Technology Co., Ltd. (Chengdu, China). Fructose (21), glucose (22), and sucrose (23) were purchased from Spring and Autumn Biological Engineering Co., Ltd. (Nanjing, China), and the stachyose (24) was purchased from Chroma-Biotechnology Co., Ltd. (Chengdu, China). The purity of each compound was over 98\%, as determined by HPLC analysis.

\subsection{Plant Materials}

The samples were collected from the medicinal botanical garden of Nanjing University of Chinese Medicine between June 2015 to May 2016, and their botanical origins were identified by Prof. Jin-ao Duan of the Jiangsu Collaborative Innovation Center of Chinese Medicinal Resources Industrialization, Nanjing University of Chinese Medicine, Nanjing, China. The samples were separated into different parts as roots, leaves, stems, and flowers after collection, dried at $50{ }^{\circ} \mathrm{C}$ individually, then pulverized into homogeneous powders (40 mesh) and stored dry at room temperature before analysis. The voucher specimens were deposited in the Herbarium of the Nanjing University of Chinese Medicine. Information about these samples is summarized in Table 5. 
Table 5. Harvest time, growth period, and harvest parts of S. miltiorrhiza.

\begin{tabular}{|c|c|c|c|}
\hline $\begin{array}{l}\text { Collecting Time } \\
\text { (Day Month Year) }\end{array}$ & Growth Stage & Phenological Phase & Harvest Parts \\
\hline 1 April 2016 & \multirow{4}{*}{ Turn green } & Leaf expansion period & Roots, Leaves \\
\hline 29 April 2016 & & \multirow{2}{*}{ Early bloom stage } & Roots, Stems, Leaves, Flowers \\
\hline 14 May 2016 & & & Roots, Stems, Leaves, Flowers \\
\hline 30 May 2016 & & Full-bloom stage & Roots, Stems, Leaves, Flowers \\
\hline 25 June 2015 & reproductive growth of the & \multirow{2}{*}{ Late bloom stage } & Roots, Stems, Leaves, Flowers \\
\hline 4 July 2016 & aerial parts & & Roots, Stems, Leaves \\
\hline 14 July 2015 & & \multirow[t]{2}{*}{ Branch growth period } & Roots, Stems, Leaves \\
\hline 23 July 2015 & & & Roots, Stems, Leaves \\
\hline 12 August 2015 & & \multirow{2}{*}{ Form stable phase of the aerial parts } & Roots, Stems, Leaves \\
\hline 26 August 2015 & & & Roots, Stems, Leaves \\
\hline
\end{tabular}

\subsection{Preparation of Standard Solutions}

3.3.1. Standard Solution Preparation of Salvianolic Acids, Flavonoids, Tanshinones, and Triterpenes

A mixed standard stock solution containing the reference compounds 1-20 was prepared in $90 \%$ methanol, and the concentration for the 20 analytes were as follows: $0.229,0.162,0.190,0.202$, $0.185,0.148,0.253,0.190,0.257,0.174,0.071,0.126,0.236,0.186,0.107,0.113,0.089,0.144,0.176$, and $0.207 \mathrm{mg} / \mathrm{mL}$. Working standard solutions for calibration curves were prepared by diluting the mixed standard stock solution with $80 \%$ methanol to different concentrations.

\subsubsection{Standard Solution Preparation of Saccharides}

The mixed standard stock solution containing the reference compounds 21-24 was prepared with Millipore water at concentrations of 3.20,3.05, 2.61, and $5.03 \mathrm{mg} / \mathrm{mL}$, then diluted to different concentrations. All of the solutions were stored in a refrigerator at $4{ }^{\circ} \mathrm{C}$ until use and filtered through a $0.22 \mu \mathrm{m}$ cellulose membrane before analysis.

\subsection{Preparation of Sample Solutions}

3.4.1. Sample Solutions Preparation for Salvianolic Acids, Flavonoids, Tanshinones, and Triterpenes Analysis

The dried powder $(0.5 \mathrm{~g})$ from various parts of $S$. miltiorrhiza during different harvest times, which was weighed accurately, was put into a $50 \mathrm{~mL}$ conical flask with a stopper, and $50 \mathrm{~mL} \mathrm{80 \%} \mathrm{methanol}$ was added. After accurate weighing, ultrasonication $(100 \mathrm{kHz})$ was performed at $50{ }^{\circ} \mathrm{C}$ for $45 \mathrm{~min}$; afterwards the same solvent was added to compensate for the weight lost during extraction.

\subsubsection{Sample Solutions Preparation for Saccharides Analysis}

As above, the dried powder $(0.5 \mathrm{~g})$ was weighed accurately and $50 \mathrm{~mL}$ ultrapure water was added. After accurate weighing, reflux extraction was performed for $1 \mathrm{~h}$ and compensation for the weight lost with ultrapure water was conducted. After centrifugation $(13,000 \mathrm{rpm}, 10 \mathrm{~min})$ and filtering through a $0.22 \mu \mathrm{m}$ membrane filter, all of the sample solutions were stored at $4{ }^{\circ} \mathrm{C}$ before the injection into UPLC-TQ-MS/MS and HPLC-ELSD systems for analysis. 


\subsection{Chromatographic Conditions and Instrumentation}

3.5.1. Analysis for Salvianolic Acids, Flavonoids, Tanshinones, and Triterpenes.

Analysis was performed on a Waters Acquity UPLC system (Waters, Milford, MA, USA), which consists of a quaternary pump solvent management system, an online degasser, an autosampler, and a triple quadrupole mass detector. An Acquity UPLC BEH C 18 (2.1 mm $\times 100 \mathrm{~mm}, 1.7 \mu \mathrm{m})$ column was applied for all analyses. The mobile phase was composed of A (water and $0.1 \%$ formic acid) and B (acetonitrile) using a gradient elution of $3 \% \mathrm{~B}$ at $0-1 \mathrm{~min}, 3-30 \% \mathrm{~B}$ at $1-6 \mathrm{~min}, 30-40 \% \mathrm{~B}$ at 6-7 $\mathrm{min}, 40-95 \% \mathrm{~B}$ at 7-10 $\mathrm{min}$, and $95-95 \% \mathrm{~B}$ at $10-12 \mathrm{~min}$. The flow rate of the mobile phase was set at $0.40 \mathrm{~mL} / \mathrm{min}$. The column temperature was conditioned at $35{ }^{\circ} \mathrm{C}$, the autosampler was maintained at $4{ }^{\circ} \mathrm{C}$, and the injection volume was $2 \mu \mathrm{L}$.

The triple quadrupole (TQ) mass spectrometer was operated in both positive and negative modes with a capillary voltage of $3 \mathrm{kV}$, a cone gas flow of $20 \mathrm{~L} / \mathrm{h}$, a collision gas flow of $0.15 \mathrm{~mL} / \mathrm{min}$, a desolvation gas flow of $1000 \mathrm{~L} / \mathrm{h}$, a desolvation temperature of $550{ }^{\circ} \mathrm{C}$, a source temperature of $150^{\circ} \mathrm{C}$, and full-scan spectra from 100 to $1000 \mathrm{Da}$. The raw data were acquired and processed with MassLynx 4.1 software (Waters Corporation, Milford, MA, USA).

\subsubsection{Analysis for Saccharides}

The HPLC-ELSD conditions were used for the determination of the saccharides, a Waters Alliance 2695 liquid chromatograph system (Waters, Milford, MA, USA) equipped with an Alltech 2000 evaporative light-scattering detector (Grace, Deerfield, MA, USA) was used. The chromatographic separations were performed over a Prevail Carbohydrate ES $(250 \mathrm{~mm} \times 4.6 \mathrm{~mm}, 5 \mu \mathrm{m})$ column at a column temperature of $35^{\circ} \mathrm{C}$. The column was eluted with a mixture of acetonitrile (mobile phase A) and water (mobile phase $\mathrm{B}$ ) at a flow rate of $1.0 \mathrm{~mL} / \mathrm{min}$. The elution conditions were as follows: 0-23 $\mathrm{min}, 20-50 \% \mathrm{~B} ; 23-26 \mathrm{~min}$, and 50-20\% B. The drift tube temperature of the ELSD was set at $80^{\circ} \mathrm{C}$, and using nitrogen as the carrier gas at a flow rate set at $3.0 \mathrm{~L} / \mathrm{min}$, the gain value was 10 , and the injection volume was $10 \mu \mathrm{L}$ for roots and leaves analysis, and $20 \mu \mathrm{L}$ for stems and flowers analysis.

\subsection{Validation of the Methods}

\subsubsection{Calibration Curves, LODs, and LOQs}

The linearity was obtained by preparing a series of concentrations of standard solution with at least five appropriate concentrations in duplicate. The lowest concentration of the working solution for calibration use was diluted with the corresponding solvent to a series of concentrations. LODs and LOQs for each analyte were acquired while the $S / N$ was 3 and 10, respectively.

\subsubsection{Precision, Repeatability, and Stability}

To evaluate the precision, we analyzed the standard solutions with six replicates, and the RSD of the peak area for each standard compounds was calculated. To confirm the repeatability in this developed method, six different sample solutions were prepared from the same sample (sample Y1, the leaves of SM which were collected on 8 June 2015) were analyzed and variations were expressed by RSD. The stability was evaluated by storing the sample solutions mentioned above (Y1) at $25^{\circ} \mathrm{C}$, then analyzed at $0,2,4,8,12$, and $24 \mathrm{~h}$, respectively.

\subsubsection{Recovery}

A spike recovery test was used to evaluate the accuracy of these methods, which was performed by adding the corresponding compounds at high (120\%), medium (100\%), and low (80\%) levels in the sample (Y1) preparation, then measured in six duplicates. The spiked samples were then extracted, 
processed, and quantified in accordance with the methods mentioned above. The spike recoveries were calculated using the following equation:

Recovery $\%=[($ measured amount - original amount $) /$ amount added $] \times 100 \%$.

\subsection{Sample Determination}

All of the samples were prepared according to Section 3.4, and determined thereafter according to the Section 3.5 chromatographic conditions and instrumentation. Quantification of each compound was performed on the basis of linear calibration plots of the peak areas versus the corresponding concentration. The content of total phenolic acids was the sum of danshensu, protocatechuic aldehyde, caffeic acid, rosmarinic acid, lithospermic acid, salvianolic acid B, and salvianolic acid A. The sum of rutin, isoquercitrin, and astragalin defined the total flavonoids. Total tanshinones was defined as the sum of tanshinone IIB, dihydrotanshinone I, cryptotanshinone, neocryptotanshione, tanshinone I, tanshinone IIA, miltiradiene and miltirone. The total triterpenes included oleanolic acid and ursolic acid.

\section{Conclusions}

In the present study, the simple and reliable UPLC-TQ-MS/MS and HPLC-ELSD analytical methods for rapidly quantifying multiple components in S. miltiorrhiza was established. As a result, 24 components were identified and quantified, which clearly suggested the aerial parts of S. miltiorrhiza contain phenolic acids, flavonoids, triterpenes, and saccharides, without the liposoluble tanshinones being detected. Therefore, the aerial parts of S. miltiorrhiza could be a promising natural source for phenolic acids and flavonoids. During the planting production of TCM, suitable harvest time is crucial for the yield and quality of medicinal materials. To the authors' knowledge, this is the first report about distribution and dynamic changes of chemical constituents in various parts of S. miltiorrhiza during different harvest seasons. Apart from the difference of chemical composition between aboveground and underground parts of S. miltiorrhiza, the data provided an effective reference for the optimal harvest time selection according to the accumulation dynamics of target components. It recommended that the optimal harvest time for the roots and rhizomes of S. miltiorrhiza is the seedling stage in spring and, for the aerial parts of S. miltiorrhiza, is July to August. The research could provide the theoretical basis and scientific evidence for comprehensive development and utilization of S. miltiorrhiza resources.

Since all of the samples were collected from the medicinal botanical garden of Nanjing University of Chinese Medicine, dynamic accumulation of chemical constituents in various parts of S. miltiorrhiza during different growth periods from other geographical areas is unclear, which remains of interest for further research. Nevertheless, the results and established methods of this paper were intended to provide a favorable reference for the study of S. miltiorrhiza from other origins. Furthermore, the established UPLC-TQ-MS/MS and HPLC-ELSD methods can also be applied in future research of S. miltiorrhiza and its preparation of medicines.

Supplementary Materials: Supplementary materials are available online.

Acknowledgments: This work was supported by the National Natural Science Foundation of China (No. 81673533; $81373889 ; 81473408$ ) and supported by program for excellent talents in school of pharmacy of Nanjing university of Chinese Medicine (15ZYXET-2). This work was also supported by the Construction Project for Jiangsu Key Laboratory for High Technology Research of TCM Formulae (BM2010576; BK2010561), and a project funded by the Priority Academic Program Development of Jiangsu Higher Education Institutions (ysxk-2014). This work also supported by the 2013 Program for New Century Excellent Talents by the Ministry of Education (Grant NCET-13-0873), 333 High-level Talents Training Project Funded by Jiangsu Province, and Six Talents Project Funded by Jiangsu Province (2012-YY-010).

Author Contributions: The list authors contributed to this work as follows: S.S. and J.D. conceived and designed the experiments, and H.Z. wrote the paper; H.Z., X.X., and X.S. performed the experiment and analyzed the data; Z.Z., Y.W., S.G., and H.Y. contributed reagents/materials/analysis tools; and S.S. and D.Q. acquired funding for the research.

Conflicts of Interest: The authors declare no conflict of interest. 


\section{References}

1. Yang, L.; Ding, G.H.; Lin, H.Y.; Cheng, H.N.; Kong, Y.; Wei, Y.K.; Fang, X.; Liu, R.Y.; Wang, L.J.; Chen, X.Y.; et al. Transcriptome analysis of medicinal plant Salvia miltiorrhiza and identification of genes related to tanshinone biosynthesis. PLoS ONE 2013, 8, e80464. [CrossRef]

2. Cai, H.D.; Su, S.L.; Li, Y.H.; Zhu, Z.H.; Guo, J.M.; Zhu, Y.; Guo, S.; Qian, D.W.; Duan, J.A. Simultaneous determination of four tanshinones by UPLC-TQ/MS and their pharmacokinetic application after administration of single ethanol extract of danshen combined with water extract in normal and adenine-induced chronic renal failure rats. Molecules 2016, 21, 1630. [CrossRef] [PubMed]

3. Li, Y.G.; Song, L.; Liu, M.; Hu, Z.B.; Wang, Z.T. Advancement in analysis of Salviae miltiorrhizae Radix et Rhizoma (Danshen). J. Chromatogr. A 2009, 1216, 1941-1953. [CrossRef] [PubMed]

4. Zhang, J.; An, S.J.; Fu, J.Q.; Liu, P.; Shao, T.M.; Li, M.; Li, X.; Jiao, Z.; Chai, X.Q. Mixed aqueous extract of Salvia miltiorrhiza reduces blood pressure through inhibition of vascular remodelling and oxidative stress in spontaneously hypertensive rats. Cell. Physiol. Biochem. 2016, 40, 347-360. [CrossRef] [PubMed]

5. Jiang, Y.Y.; Wang, L.; Zhang, L.; Wang, T.; Yu, L.; Ding, C.B.; Yang, R.W.; Wang, X.L.; Zhou, Y.H. Characterization, antioxidant and antitumor activities of polysaccharides from Salvia miltiorrhiza Bunge. Int. J. Biol. Macromol. 2014, 70, 92-99. [CrossRef] [PubMed]

6. Su, C.Y.; Qian, L.M.; Rahman, K.; Han, T.; Qin, L.P. Salvia miltiorrhiza: Traditional medicinal uses, chemistry, and pharmacology. Chin. J. Nat. Med. 2015, 13, 163-182. [CrossRef]

7. Ma, S.L.; Zhang, D.W.; Lou, H.X.; Sun, L.R.; Ji, J.B. Evaluation of the anti-inflammatory activities of tanshinones isolated from Salvia miltiorrhiza var. Alba roots in THP-1 macrophages. J. Ethnopharmacol. 2016, 188, 193-199. [CrossRef] [PubMed]

8. Hu, B.; Amin, R.; Kumar, S.; Kunaparaju, N.; Graham, S.M. Bioassay-guided isolation of the antidiabetic active principle from Salvia miltiorrhiza and its stimulatory effects on glucose uptake using 3T3-L1 adipocytes. Med. Chem. 2014, 4, 592-597.

9. Chen, X.P.; Guo, J.J.; Bao, J.L.; Lu, J.J.; Wang, Y.T. The anticancer properties of Salvia miltiorrhiza Bunge (Danshen): A systematic review. Med. Res. Rev. 2014, 34, 768-794. [CrossRef] [PubMed]

10. Jiang, Y.Y.; Zhang, L.; Rupasinghe, H.P. Antiproliferative effects of extracts from Salvia officinalis L. and Saliva miltiorrhiza Bunge on hepatocellular carcinoma cells. Biomed. Pharmacother. 2017, 85, 57-67. [CrossRef] [PubMed]

11. Luo, H.L.; Kong, W.J.; Hu, Y.C.; Chen, P.; Wu, X.R.; Wan, L.; Yang, M.H. Quality evaluation of Salvia miltiorrhiza Bge. by ultra high performance liquid chromatography with photodiode array detection and chemical fingerprinting coupled with chemometric analysis. J. Sep. Sci. 2015, 38, 1544-1551. [CrossRef] [PubMed]

12. Jing, W.G.; Zhang, J.; Zhang, L.Y.; Wang, D.Z.; Wang, Y.S.; Liu, A. Application of a Rapid and Efficient Quantitative Analysis Method for Traditional Chinese Medicines: The Case Study of Quality Assessment of Salvia miltiorrhiza Bunge. Molecules 2013, 18, 6919-6935. [CrossRef] [PubMed]

13. Zhou, G.J.; Wang, W.; Xie, X.M.; Qin, M.J.; Kuai, B.K.; Zhou, T.S. Post-Harvest induced production of salvianolic acids and significant promotion of antioxidant properties in roots of Salvia miltiorrhiza (Danshen). Molecules 2014, 19, 7207-7222. [CrossRef] [PubMed]

14. Liu, M.; Li, Y.G.; Zhang, F.; Yang, L.; Chou, G.X.; Wang, Z.T.; Hu, Z.B. Chromatographic fingerprinting analysis of Danshen root (Salvia miltiorrhiza Radix et Rhizoma) and its preparations using high performance liquid chromatography with diode array detection and electrospray mass spectrometry (HPLC-DAD-ESI/MS). J. Sep. Sci. 2007, 30, 2256-2267. [CrossRef] [PubMed]

15. Pang, H.Q.; Wu, L.; Tang, Y.P.; Zhou, G.S.; Qu, C.; Duan, J.A. Chemical analysis of the herbal medicine Salviae Miltiorrhizae Radix et Rhizoma (Danshen). Molecules 2016, 21, 51. [CrossRef] [PubMed]

16. Duan, J.A. Resources Utilization of Chinese Herbal Medicine Wastes; Chemical Industry Press: Beijing, China, 2013; pp. 427-431.

17. Duan, J.A. Resources Chemistry of Chinese Medicinal Materials: Theoretical Basis and Resource Recycling Utilization; Science Press: Beijing, China, 2015; pp. 459-473.

18. Hang, L. Studies on the Chemical Constituents from Aerial Parts of Salvia miltiorrhiza Bunge. Master's Thesis, Northwest University of Science and Technology, Xi'an, China, 2008. 
19. Zhang, Y.; Li, X.; Wang, Z.Z. Antioxidant activities of leaf extract of Salvia miltiorrhiza Bunge and related phenolic constituents. Food Chem. Toxicol. 2010, 48, 2656-2662. [CrossRef] [PubMed]

20. Zhang, Q.; Chang, Z.; Yang, J.; Wang, Q. Antiatherogenic property of triterpenoids-enriched extract from the aerial parts of Salvia miltiorrhiza. Phytother. Res. 2008, 22, 1040-1045. [CrossRef] [PubMed]

21. Leng, J.; Chen, M.H.; Zhou, Z.H.; Lu, Y.W.; Wen, X.D.; Yang, J. Triterpenoids-enriched extract from the aerial parts of Salvia miltiorrhiza regulates macrophage polarization and ameliorates insulin resistance in high-fat fed mice. Phytother. Res. 2017, 31, 100-107. [CrossRef] [PubMed]

22. Zhang, H.; Jia, M.; Yang, Y. Screening anti-cerebral ischemia effective composition of aerial part of Salvia. Pharmacol. Clin. Chin. Mater. Med. 2013, 29, 133-136.

23. Li, X.; Wang, Z.Z. Chemical composition, antimicrobial and antioxidant activities of the essential oil in leaves of Salvia miltiorrhiza Bunge. J. Essent. Oil Res. 2009, 21, 476-480. [CrossRef]

24. Chen, H.; Zhang, Q.; Wang, X.M.; Yang, J.; Wang, Q. Qualitative analysis and simultaneous quantification of phenolic compounds in the aerial parts of Salvia miltiorrhiza by HPLC-DAD and ESI/MS(n). Phytochem. Anal. 2011, 22, 247-257. [CrossRef] [PubMed]

25. Shi, G.Y.; Guo, Q.M.; Zhou, F.Q. Study on chemical constituents of leaves of Salvia miltiorrhiza Bge. J. Shanxi Univ. 2015, 38, 692-695.

26. Zheng, Y.F.; Ge, T.; Xia, Y.M.; Zhou, L.S.; Cui, J.; Peng, G.P. Study on dynamic change of stachyose of Salvia miltiorrhiza at different parts in growing period. J. Nanjing Univ. Tradit. Chin. Med. 2014, 30, 573-575.

27. Zhao, Z.G.; Gao, S.R.; Xie, J.; Yan, B.B.; Hou, J.L.; Wang, W.Q.; Song, Y.; Zhang, X.M.; Li, J. Dynamic study on biomass and bioactive ingredients accumulation of roots of Salvia miltiorrhizae in different growth periods. Mod. Chin. Med. 2015, 15, 1171-1176.

28. Liu, H.Y.; Wang, X.D.; Wang, D.H.; Zou, Z.R.; Liang, Z.S. Effect of drought stress on growth and accumulation of active constituents in Salvia miltiorrhiza Bunge. Ind. Crop. Prod. 2011, 33, 84-88. [CrossRef]

29. Sha, X.X.; Dai, X.X.; Su, S.L.; Yan, H.; Guo, S.; Qian, D.W.; Tang, Z.S.; Duan, J.A. Quality standard for stems and leaves of Salvia miltiorrhiza. Chin. J. Pharm. Anal. 2016, 36, 1096-1100.

30. Sha, X.X.; Su, S.L.; Shen, F.; Jiang, S.; Yan, H.; Guo, S.; Qian, D.W.; Duan, J.A. Distribution of salvianolic acids in aerial parts of Salvia miltiorrhiza during different growing periods and accumulation dynamic analysis. Chin. Tradit. Herb. Drugs 2015, 46, 3414-3419.

31. Li, J.T. Study on Histochemical Localization and Transport of Secondary Metabolism in Salvia miltiorrhiza Bunge. Master's Thesis, Northwest University of Science and Technology, Xi'an, China, 2008.

32. Tain, L.; Zeiger, E. Plant Physiology, 5th ed.; Sinauer Associates, Inc.: Sunderland, MA, USA, 2010; pp. $179-189$.

33. Li, T.; Lu, X.S.; Yang, X.B. Evaluation of clinical safety and beneficial effects of stachyose-enriched $\alpha$-galacto-oligosaccharides on gut microbiota and bowel function in humans. Food Funct. 2017, 8, $262-269$. [CrossRef] [PubMed]

34. Chen, Y.H.; Guo, Q.S.; Zhu, Z.B.; Zhang, L.X. Changes in bioactive components related to the harvest time from the spices of Prunella vulgaris. Pharm. Biol. 2012, 50, 1118-1122. [CrossRef] [PubMed]

35. Du, Q.H.; Zhang, Q.Y.; Han, T.; Jiang, Y.P.; Peng, C.; Xin, H.L. Dynamic changes of flavonoids in Actinidia valvata leaves at different growing stages measured by HPLC-MS/MS. Chin. J. Nat. Med. 2016, 14, 66-72. [PubMed]

Sample Availability: Samples of the roots, stems, leaves and flowers of Salvia miltiorrhiza and compounds including danshensu, protocatechuic aldehyde, caffeic acid, rosmarinic acid, lithospermic acid, salvianolic acid B, salvianolic acid A, rutin, isoquercitrin, astragalin, tanshinone IIB, dihydrotanshinone I, cryptotanshinone, neocryptotanshione, tanshinone I, tanshinone IIA, miltiradiene, miltirone, oleanolic acid, ursolic acid, fructose, glucose, sucrose, stachyose are available from the authors.

(C) 2017 by the authors. Licensee MDPI, Basel, Switzerland. This article is an open access article distributed under the terms and conditions of the Creative Commons Attribution (CC BY) license (http:/ / creativecommons.org/licenses/by/4.0/). 